\title{
The MAGICA Type Inference Engine for MATLAB ${ }^{\circledR \star}$
}

\author{
Pramod G. Joisha and Prithviraj Banerjee \\ Department of Electrical and Computer Engineering, Northwestern University, USA. \\ \{pjoisha, banerjee\}@ece.northwestern.edu
}

\section{Introduction}

MAGICA (MAthematica system for General-purpose Inferring and Compile-time Analyses) is an extensible inference engine that can determine the types (value range, intrinsic type and array shape) of expressions in a MATLAB program. Written as a Mathematica application, it is designed as an add-on module that any MATLAB compiler infrastructure can use to obtain high-quality type inferences.

\subsection{A Type Inference Using MAGICA}

Lines In [1] and Out [2] below demonstrate a simple interaction with MAGICA through a notebook interface 1 On line In [1], the MAGICA type function object is applied on a representation of the MATLAB expression $\tanh (3.78 i)$. MAGICA's response, shown on Out [2], is the inferred type of $\tanh (3.78 i)$. In this case, "type" is the expression $\{v, i, s\}$ where $v, i$ and $s$ are the value range, intrinsic type and array shape of $\tanh (3.78 i)$; Out [2] indicates these to be the point $0.742071 \mathrm{i}$, the $\$$ nonreal intrinsic type designator, and the twodimensional array shape with unit extents along both dimensions - that is, the scalar shape.

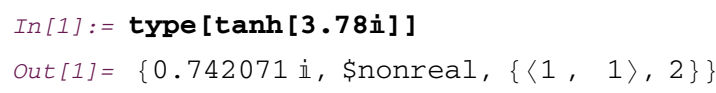

\subsection{Feature Support}

The above is an example of a type inference on a single MATLAB expression. MAGICA can infer the types of whole MATLAB programs comprising an arbitrary number of user-defined functions, each having an arbitrary number of statements. User-defined functions can return multiple values, can consist of

* This research was supported by DARPA under Contract F30602-98-2-0144, and by NASA under Contract 276685/NAS5-00212. Mathematica ${ }^{\circledR}$ fonts by Wolfram Research, Inc.

1 The outputs in this paper can be exactly reproduced by typing the code shown against each $\operatorname{In}[n]:=$ prompt into a notebook interface to version 1.0 of MAGICA, running on Mathematica 4.1 .

G. Hedin (Ed.): CC 2003, LNCS 2622, pp. 121125 2003.

(C) Springer-Verlag Berlin Heidelberg 2003 
assignment statements, the for and while loops, and the if conditional statement. (All these MATLAB constructs are explained in [3.) In addition, MAGICA can handle close to 70 built-in functions in MATLAB. These include important Type II operation 2 like subsref, subsasgn and colon that are used in array indexing and colon expressions. For the most part, the full or nearly the full semantics of a built-in function, as specified in [3], is supported. For instance, subscripts in array indexing expressions can themselves be arrays, and arrays can be complex-valued. Not all of MATLAB's features are currently handled; these include structures, cell arrays and recent additions like function handles.

\section{Representing MATLAB in MAGICA}

MAGICA symbolically represents constructs in MATLAB. An example of this is the Mathematica expression plus [a, b], which is MAGICA's representation of the MATLAB expression $\mathrm{a}+\mathrm{b}$. On line In [1] above, the Mathematica expression tanh [3.781i] was used to denote the MATLAB expression tanh(3.78i). The idea of functionally representing a MATLAB expression can also be used to denote high-level constructs. For instance, the MATLAB assignment statement $1 \leftarrow \cos (3.099)$, where 1 is a MATLAB program variable, is represented in MAGICA as shown on line In [2] below.

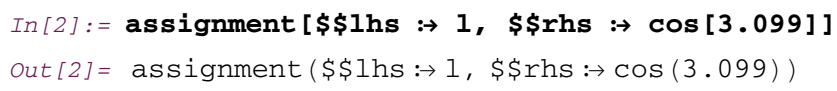

The expression's head is assignment and this is used to uniquely identify MATLAB assignments. The tags $\$$ \$ 1 hs and $\$$ \$rhs serve to identify the assignment's left-hand side and right-hand side. We call 1 and cos [3.099] as tag values. A tag value can be any expression; this allows for the representation of arbitrary MATLAB assignments, including the multiple-value assignment [3].

In general, MATLAB statements are represented in MAGICA as

$$
h\left[x_{1}: \rightarrow y_{1}, x_{2}: \rightarrow y_{2}, \ldots, x_{n}: \rightarrow y_{n}\right]
$$

where the head $h$ serves as a construct identifier, and where the delayed rules [4] $x_{i}: \rightarrow y_{i}(1 \leq i \leq n)$ stand for tag-value pairs. MAGICA places no significance on the position of a tag-value pair; this point should be kept in mind when making new definitions to extend the MAGICA system. A fair amount of documentation regarding data structure layouts has been coded into MAGICA itself as usage messages [4]; this provides a convenient, on-line way of pulling up layout information while interacting with MAGICA.

$\overline{2}$ MATLAB's built-in functions can be classified into one of three groups, based on how the shapes of the outputs are dependent on the shapes of the inputs [1]. Type I built-ins produce outputs whose shapes are completely determined by the shapes of the arguments, if any. Type II built-ins produce an output whose shape is also dependent on the elemental values of at least one input. All remaining built-ins fall into the Type III group. 
In $[3]:=$ ?if

if $\$ \$$ condition : $>c_{-}, \$ \$$ then $:>t_{-}, \$ \$$ else $\left.:>e_{-}\right]$

is the functional equivalent of an if statement in MATLAB. Forms such as

\$ $\$$ condition $\rightarrow \mathrm{c}, \$$ \$then $\rightarrow \mathrm{t}$ and $\$$ \$else $\rightarrow \mathrm{e}$ can also be used.

\section{Transitive Closure of a Graph}

The two boxes in Figure 1 display a complete MATLAB program that computes the transitive closure of a graph. The graph is represented in the $N \times N$ adjacency matrix A, which is initialized arbitrarily in the function tclosure. Its transitive closure is returned in B. The shown code is directly from Alexey Malishevsky's thesis [2], with three nontrivial changes: (1) the tic and toc timing commands were removed, (2) disp was used to display B, and (3) the original monolithic script was reorganized into two files, one containing the function driver and the other containing tclosure.

\subsection{M-File Contexts}

Input files that constitute a MATLAB program are referred to as $M$-files in MATLAB parlance. Every M-file has its own parsed representation in MAGICA, which we call an M-file context. Through Mathematica's information-hiding context mechanism [4, MAGICA provides a way to save, and later retrieve, the M-file contexts of a MATLAB program. On line In [4] below, the M-file contexts of the two user-defined functions driver and tclosure, saved in an earlier session of MAGICA, are loaded from disk 3

$$
\operatorname{In}[4]:=\operatorname{Scan}[1 \text { ad [\#, load\$Disk } \rightarrow \text { True] \&, \{"tclosure'", "driver'"\}] }
$$

An M-file context is basically a collection of Mathematica definitions that capture information about a user-defined MATLAB function. As an example, for a user-defined function $f$, a definition is made against the statements function object so that statements [f] expands to the function body of $f$. This is how the type object operates on the statements in the body of driver on line In [5] below 4

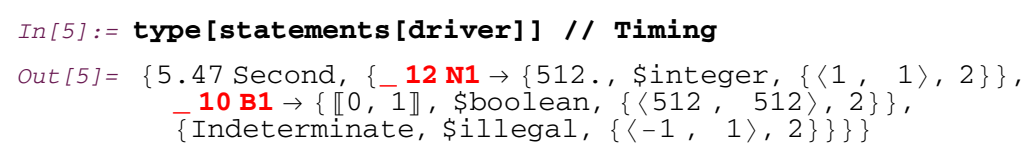

\footnotetext{
3 These representations are in ASCII, and can be manually or automatically generated.

${ }^{4}$ The timings are on a $440 \mathrm{MHz}$ Solaris 7 UltraSPARC-IIi having $128 \mathrm{MB}$ of main memory.
} 
$\S 3$ were produced in advance. Figure 2 also shows the disk image of a sample M-file context.

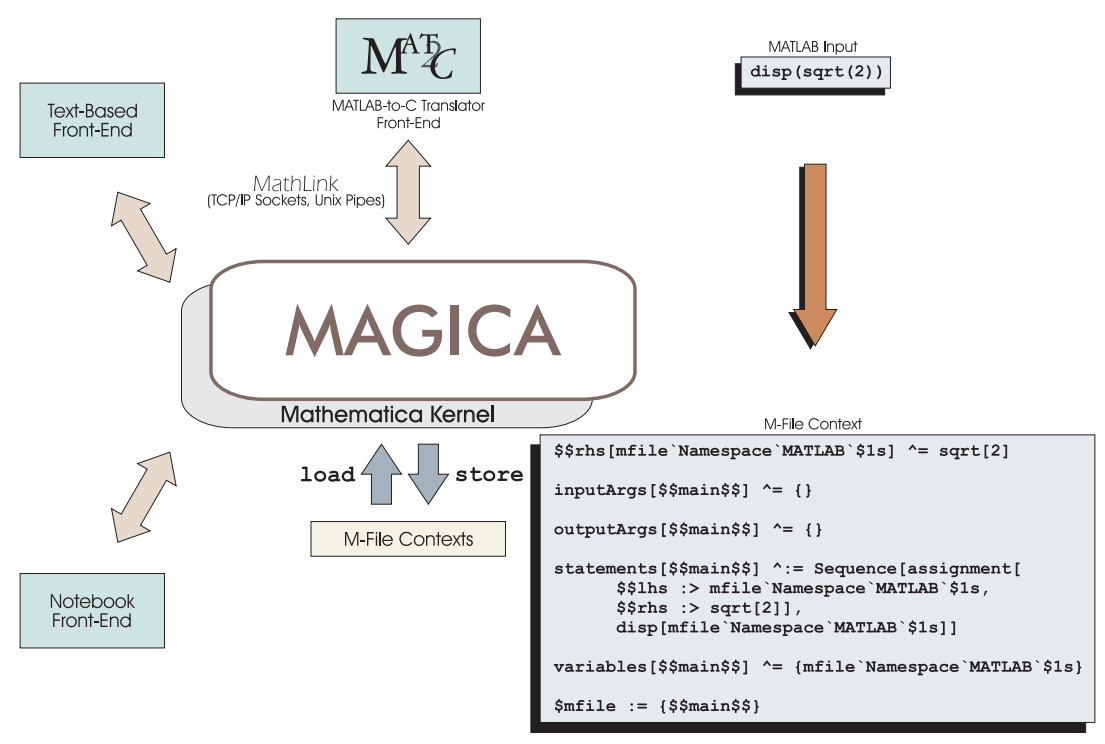

Fig. 2. The MAGICA Architecture

\section{Summary}

This paper briefly introduced a software tool called MAGICA that infers value ranges, intrinsic types and array shapes for the MATLAB programming language. Though shown in an interactive mode in this paper, MAGICA can also be used in a batch mode from a custom front-end. Currently, MAGICA is being used this way by $\mathbf{M}^{\mathrm{A}} \mathrm{C}$, a MATLAB-to-C translator that converts a MATLAB source to optimized $\mathrm{C}$ code.

\section{References}

1. P.G. Joisha, U.N. Shenoy, ad P. Banerjee. "An Approch to Array Shape Determination in MATLAB". Technical report CPDC-TR-2000-10-010, Department of Electrical and Computer Engineering, Northwestern University, October 2000.

2. A. Malishevsky. "Implementing a Run-Time Library for a Parallel MATLAB Compiler". M.S. report, Oregon State University, April 1998.

3. The MathWorks, Inc. MATLAB: The Language of Technical Computing, January 1997. Using MATLAB (Version 5).

4. S. Wolfram. The Mathematica Book, 4th ed. Wolfram Media, Inc., 1999. 